\title{
Measuring projection tachistoscope shutter characteristics
}

\author{
ROBERT MADIGAN and SUSAN JOHNSON \\ University of Alaska, Anchorage, Alaska
}

\begin{abstract}
Projection tachistoscopes have shutters that control the presentation of stimuli. Shutter characteristics such as opening times, closing times, and mechanical reliability are important factors in applications of these tachistoscopes. The device described in this report allows the operating characteristics of shutters used in projection tachistoscopes to be evaluated. The use of the device is illustrated by measurements of three commercially available tachistoscope shutters. Each shutter was characterized by five parameters: opening and closing speeds, the lag between shutter activation and operation, the briefest stimulus presentation that the shutter allowed, and the shutter's ability to repeat exposure durations accurately.
\end{abstract}

Contemporary tachistoscopes are based on three proven designs. One common design involves flash tubes that illuminate stimuli placed in a darkened chamber (Bohlander, 1979). Another approach relies on cathode-ray tubes (CRTs) to present stimuli briefly (Finley, 1989). Attaching photographic shutters to slide projectors is a third altemative (Glaser, 1988). Such projection tachistoscopes are especially convenient for many research applications. Any stimulus that can be prepared as a $35-\mathrm{mm}$ slide can be presented with excellent resolution, and the projector tray can be easily controlled for changing slides.

There are, however, several concerns for the user of projection tachistoscopes. The luminance of the projection lamp changes as the bulb ages, and it must be carefully monitored. Other potential problems involve the shutters themselves, which are mechanical devices operated by electric solenoids. The shutters operate many thousands of times under the heat of a projector lamp. Frequently they are not protected from airborne dust, which can adversely affect their performance. In experiments in which short stimulus presentations are required, the shutter's mechanical reliability can be crucial. It is not uncommon for experiments to require short exposure times of $10 \mathrm{msec}$ or less (Bornstein, Leone, \& Galley, 1987; Carr, McCauley, Sperber, \& Parmelee, 1982; Marcel, 1980), so that a sticky shutter, varying 3 or $4 \mathrm{msec}$ in its presentation durations, could add significant variance to the experimental data in such situations. We have observed shutters that varied as much as $20 \mathrm{msec}$ from trial to trial in experiments in which the stimulus was programmed for a 50-msec presentation. Another potential shutter problem arises when the stimulus presentation must be precisely synchronized with a second event. Solenoid-operated shutters have a lag between the time at which the programming equipment activates the shut-

Correspondence about this article should be sent to Robert Madigan, Department of Psychology, University of Alaska, Anchorage, AK 99508. ter and the time at which the shutter responds. The lag is a function of variables such as the characteristics of the driving electronics, the reactance of the solenoid, and the mechanical condition of the shutter. This lag can cause timing aberrations in some applications.

The performance curve of a hypothetical projection tachistoscope shutter is presented in Figure 1; several critical parameters are shown. The shutter operating lags are given by $t_{1}$ and $t_{3}$; the opening and closing transition times are represented by $t_{2}$ and $t_{4}$. Parameter $t_{5}$ characterizes the stimulus presentation time.

These parameters can be measured with varying degrees of sophistication. In this article, we describe a simple device capable of measuring the basic operating characteristics of projector shutters at a level of accuracy satisfactory for most experimenters. It is useful in determining critical timing relationships, both when an experiment is set up and when shutter performance is checked periodically. After describing the device's circuit, we illustrate its use with tests performed on three common projection tachistoscope shutters.

\section{MEASURING SHUTTER CHARACTERISTICS}

The circuit of the device used to measure shutter performance is shown in Figure 2. Two outputs are provided; $\mathrm{J} 1$ is connected to a phototransistor, to allow the opening and closing characteristics of a shutter to be observed on an oscilloscope. An external timing signal can also be connected to the device at $\mathrm{J} 2$ to permit shutter exposure durations to be directly read from a frequency counter connected to $\mathrm{J} 3$.

During use, the device is positioned so that light passing through the shutter falls on phototransistor Q1. The voltage at $\mathrm{J} 1$ varies with the amount of light reaching Q1. An oscilloscope connected to J1 will show the shutter opening and closing parameters, such as $t_{2}$ and $t_{4}$ in Figure 1. Control R1 and meter M1 are used to adjust Q1's collector current, so that light from a completely 


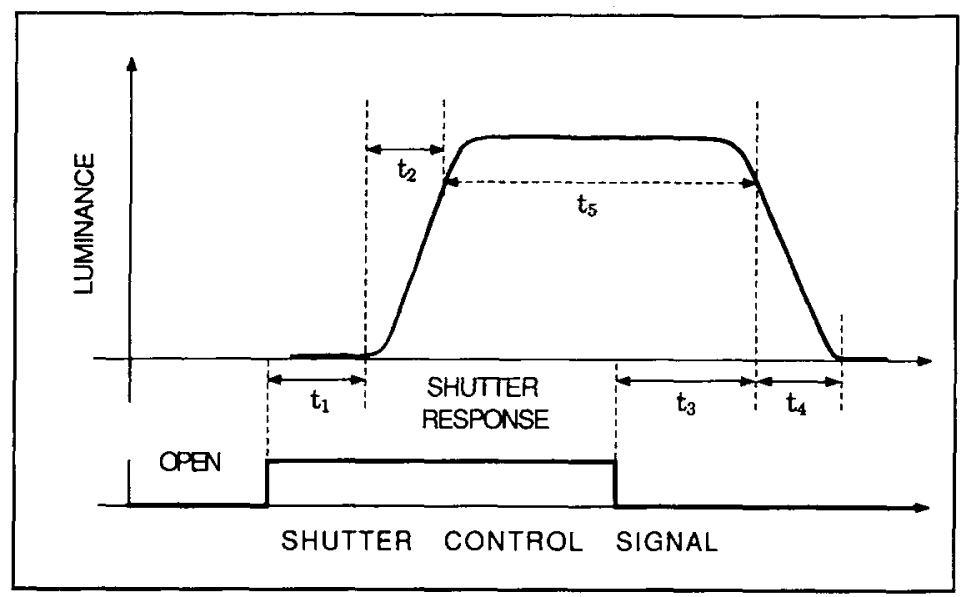

Figure 1. Operating parameters of a tachistoscope shutter. The horizontal axis represents time: $t_{1}$ and $t_{3}$ are the shutter operating lag times; $t_{2}$ and $t_{4}$ are opening and closing transition times.

open shutter almost saturates the transistor. Saturation is indicated when the voltage at $\mathrm{J} 1$ no longer shows a small amount of $60-\mathrm{Hz}$ ripple produced by the projection lamp filament as its temperature varies with the $60-\mathrm{Hz}$ line frequency. The collector current adjustment on the prototype was accomplished by setting control R1 so that meter M1 showed $0.2 \mathrm{~mA}$.
ICl is configured as a comparator and supplies a control voltage to IC2 that will gate an external signal between jacks $J 2$ and $J 3$. The values shown for $R 2$ and $R 3$ cause the gate to open when Q1's collector current is about $90 \%$ of its saturation value. We typically used a $10-\mathrm{kHz}$ sine wave at an amplitude of $1 \mathrm{~V}$ RMS. When the output at J3 was connected to a frequency counter, shutter dura-

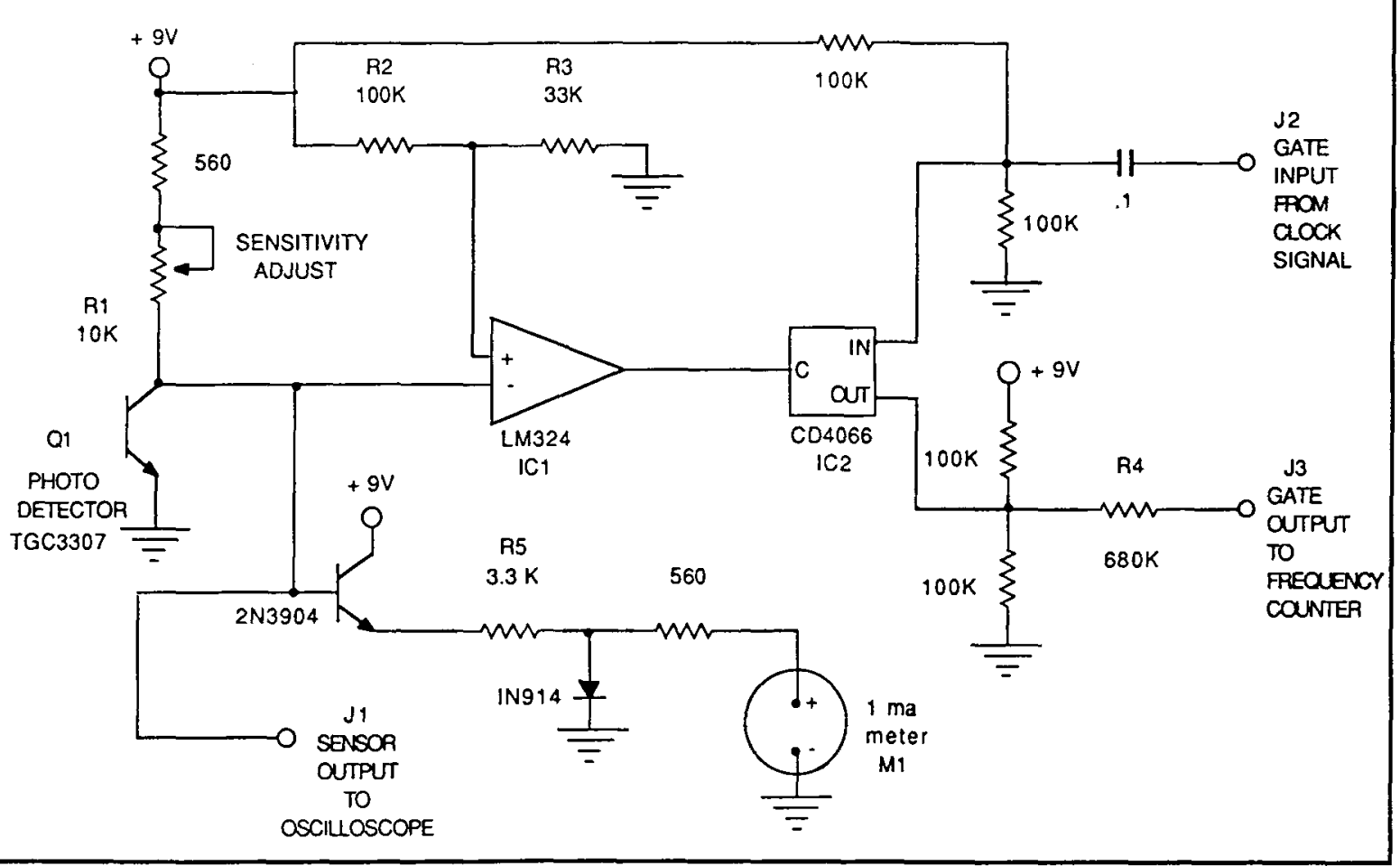

Figure 2. Circuit of a device for measuring shutter characteristics. 
tions could be measured to $0.1 \mathrm{msec}$. R4 was necessary to adjust the $10-\mathrm{kHz}$ signal voltage to a level compatible with the particular frequency counter used for the tests.

Construction of the device was uncomplicated. The components were installed in a small box $13 \times 6 \times 6 \mathrm{~cm}$, with the phototransistor mounted in a rubber grommet at a height convenient for our projection tachistoscope system. All of the parts listed in Figure 2 are readily available and inexpensive.

The output of the device at $\mathrm{J} 1$ was calibrated against a Pasco Scientific Model 8200 photometer. Calibration data are presented in Figure 3 for two levels of luminous flux corresponding to the maximum and minimum intensities that allow control RI to be properly adjusted. The device gave good representations of relative light intensities in both calibration runs.

\section{APPLICATIONS}

This device has been useful in the calibration and checking of projection tachistoscope shutters in our laboratory. To illustrate its use, in Table 1 we present data that describe the operating characteristics of three commercially available shutters (see below). Each shutter is characterized by five measurements: the opening and closing speeds, the lag between shutter activation and operation, the briefest stimulus presentation that the shutter allowed, and the shutter's ability to repeat exposure durations accurately.

\section{Method}

The three shutters tested were: a Gerbrands Model G1 166, a Lafayette Instruments Model 41010, and a Uniblitz Model 214L. ${ }^{1}$ All shutters were activated by electronic drivers supplied by their manufacturers. The Ger-

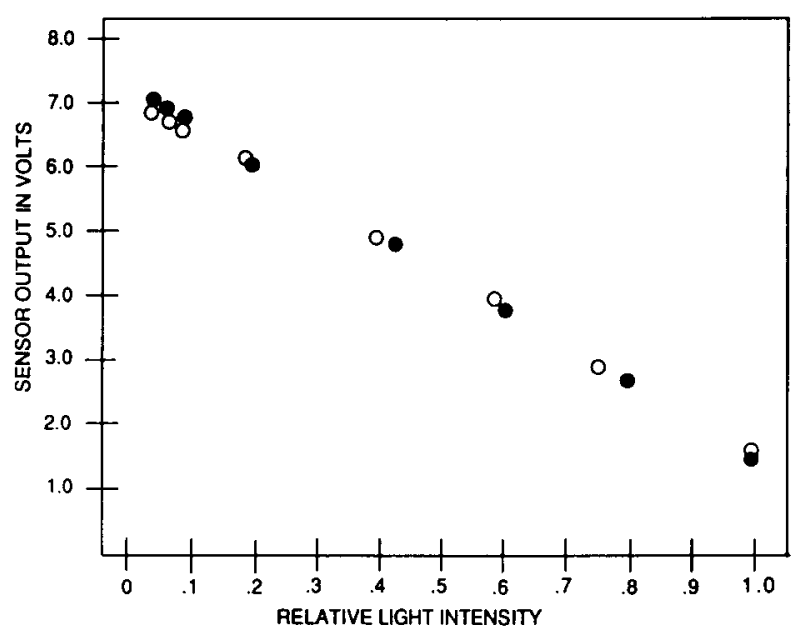

Figure 3. Response of the sensor Q1 measured at $\mathrm{J1}$ under two lighting conditions. Closed circles were obtained with a reference intensity of 1,065 lux, open circles with 112 lux. In each case, R1 was adjusted at the reference intensity so that meter M1 read $0.2 \mathrm{~mA}$; the luminance was then reduced in steps, and the voltage at $\mathrm{Jl}$ was recorded.
Table 1

Tests of Three Projection Tachistoscope Shutters

\begin{tabular}{lcccc}
\hline & \multicolumn{4}{c}{ Shutter } \\
\cline { 2 - 4 } \multicolumn{1}{c}{ Measurement } & Gerbrands & $\begin{array}{c}\text { Lafayette } \\
\text { (External) }\end{array}$ & $\begin{array}{c}\text { Lafayette } \\
\text { (Preset) }\end{array}$ & Uniblitz \\
\hline Opening time & 2.2 & 3.5 & 3.5 & 2.0 \\
Closing time & 2.0 & 4.0 & 4.0 & 3.5 \\
Operation lag & 6.0 & 12.0 & 12.0 & 3.5 \\
Minimum exposure & 3.8 & 26.0 & 7.3 & 3.5 \\
& Reliability at $10 \mathrm{msec}$ & & \\
Range of durations & 0.6 & & 3.1 & 0.4 \\
$S D$ & 0.2 & & 0.6 & 0.1 \\
& Reliability at $50 \mathrm{msec}$ & & \\
Range of durations & 0.5 & 3.0 & 4.5 & 0.4 \\
$S D$ & 0.1 & 0.6 & 1.1 & 0.1 \\
\hline
\end{tabular}

Note-All data are in milliseconds. ${ }^{*} n=30$.

brands and the Uniblitz shutters were new; the Lafayette shutter was relatively new but had seen some service. Each shutter was mounted on a standard Kodak Ektagraphic III projector. The device of Figure 1 was positioned to receive the light passed by the shutters. A computer provided the signals to control the shutters for all tests.

A number of the shutter characteristics were determined by inspection of oscilloscope records of the voltage at $\mathrm{J} l$ of Figure 1. The opening and closing times $t_{2}$ and $t_{4}$ of Figure 1) were measured from the time at which shutter movement was first detected at $\mathrm{J} 1$ until the shutter was completely open or completely closed. The operating lag was timed from the onset of the computer signal until the shutter was completely open $\left(t_{1}+t_{2}\right)$. The minimum exposure duration measured the shortest exposure time that allowed the shutter to open completely.

The shutter's reliability was determined by measuring exposure durations at J3 of Figure 1. A $10-\mathrm{kHz}$ sine wave was present at $\mathrm{J} 2$, and the device passed the signal to $\mathrm{J} 3$ when the shutter was at least $90 \%$ open. A frequency counter connected to $\mathrm{J} 3$ recorded the shutter duration. Reliability was measured at exposure durations of 10 and $50 \mathrm{msec}$ by repeatedly programming these durations and measuring the actual opening times. Thirty observations were used to determine reliability at each exposure value.

\section{Results and Discussion}

Table 1 presents the results for the three shutters evaluated. The Lafayette model showed an unexpected characteristic. The shutter can be externally activated in two different ways. One mode makes use of switch-selected exposure times $(5,10,20,50,100 \mathrm{msec}$, etc.); the other mode controls the shutter duration directly to allow arbitrary exposure durations. The preprogrammed mode produced significantly shorter exposure durations, as the table shows. However, even under the preprogrammed exposure setting, the shortest exposure time for the Lafayette shutter was $7.3 \mathrm{msec}$.

The data reveal some variability among the shutters. Although the opening times for the three shutters are almost identical, the closing times differ. All shutters are 
opened by solenoid action but closed by a return spring that pulls the shutter leaves together as the solenoid relaxes. The Gerbrands shutter showed the most symmetric opening and closing times. The activation lag times of the shutters reflect differences in their solenoids and differences in their shutter-driver electronics.

The minimum exposure durations in Table 1 show the shortest stimulus presentation achieved with each shutter. Shorter durations resulted in the shutters' not opening completely. The times reported in the table give the minimum full-open duration ( $t_{5}$ in Figure 1$)$ and do not include the opening and closing times. Both the Gerbrands shutter and the Uniblitz shutter are very close to each other and are somewhat faster than the Lafayette model operated in its preprogrammed mode. The Lafayette shutter's ability to produce very short exposure times is impaired when the exposure time is programmed externally.

The reliability of the shutters in reproducing exposure durations is also given in the table. The Uniblitz and Gerbrands shutters were almost identical in these tests. In evaluating the variance in the Lafayette exposure times, one should remember that the Lafayette shutter tested had seen some use, but that the other two shutters were new.

These data show that projection tachistoscope shutters are capable of quite acceptable performance at the stimulus exposure times typical in psychological research. However, the Lafayette shutter must be used at its preprogrammed exposure times for short-duration work. The Gerbrands and Uniblitz shutters were roughly equivalent, although the Gerbrands shutter has somewhat more symmetric opening and closing times.

The device described here could be adapted to measure the characteristics of stimulus presentations in other types of tachistoscopes by increasing the sensitivity of Q1 to light. Replacing Q1 with a phototransistor with a Darlington output, such as Motorola's MRD 370, would provide additional sensitivity. However, since the collector-toemitter saturation voltage is higher in these more sensitive Darlington transistors, R5 and R2 would both have to be changed as well. A value of $5.6 \mathrm{k} \Omega$ for $R 5$ and $68 \mathrm{k} \Omega$ for $\mathbf{R} 2$ would be reasonable starting points in the adaptation of the device for measuring stimulus presentation characteristics in flash-tube and CRT tachistoscopes.

\section{REFERENCES}

BOHLANDER, R. W. (1979). The luminance of tachistoscope lamps as a function of flash duration. Behavior Research Methods \& Instrumentation, 11, 414-418.

Bornstein, R. F., Leone, D. R., Galley, D. J. (1987). The generalizability of subliminal mere exposure effects: Influence of stimuli perceived without awareness on social behavior. Journal of Personality \& Social Psychology, 53, 1070-1079.

Carr, T. H., McCauley, C., Sperber, R. D. \& Parmelee, C. M. (1982). Words, pictures, and priming: On semantic activation, conscious identification, and the automaticity of information processing. Journal of Experimental Psychology: Human Perception \& Peformance, 8, 757-777.

FinLEY, G. P. (1989). Tachistoscope software for the Hercules display controller. Behavior Research Methods, Instruments, \& Computers. 21, 387-390.

Glaser, W. R. (1988). Technical improvements to the projection tachistoscope. Behavior Research Methods, Instruments, \& Computers, 20, 491-494.

MARCEL, T. (1980). Conscious and preconscious recognition of polysemous words: Locating the selective effects of prior verbal context. In R. S. Nickerson (Ed.), Attention and performance VIII. Hillsdale, NJ: Erlbaum.

\section{NOTE}

1. Addresses of the manufacturers are, respectively: Gerbrands Corporation, 2 Beck Road, Arlington, MA 02174; Lafayette Instrument Company, P.O. Box 5729, Lafayette, IN 47903; Vincent Associates, 1255 University Avenue, Rochester, NY 14607.

(Manuscript received July 31, 1990; revision accepted for publication December 6, 1990.) 